\title{
Constraint-Based Personalization For Business Applications
}

\author{
Kal Toth, (E-mail: ktoth@cs.orst.edu), Oregon State University
}

\begin{abstract}
This paper reports on extensions of previous work applying personalization techniques and constraint-based methods within an intelligent agent framework. The Wise Net Inc. has developed an intelligent agent framework specifically for providing advanced scalable collaborative capabilities for easy integration with existing web-enabled enterprise applications. Since the summer of 2001, the author, his colleagues, and his research assistants, have been conducting applied research aimed at discovering the desired personalization models and effects to support collaborative e-business systems. Intelligent agents are being developed to implement these personalization effects through constraint-satisfaction methods and solvers. This paper documents the approach, progress achieved to date, and future directions. This work is being supported by The Wise Net Inc., the BC Advanced Systems Institute (BC ASI), and the Canadian National Research Council (NRC) through the Industrial Research Assistance Program (IRAP).
\end{abstract}

\section{Introduction}

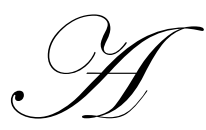

t last year's Conference, Toth and Todino [1] presented the collaboration requirements for enterprise, B2B, B2C and C2C e-business applications in a converged wired / wireless Internet context. It also described the initial intelligent agent model that was emerging at that time.

Wireless PDAs and cell phones supporting instant messaging and web access are beginning to transform the landscape. Business and messaging applications on the Internet are increasingly leveraging wireless technologies, mobility, personalization, and context to provide dynamic access to relevant information anytime, anywhere, anyhow. Personalization, context sensitivity, location, and presence information have the potential of enabling increasingly ubiquitous and pervasive collaborative computing. Of course, many difficult technical and usability challenges need to be addressed.

This paper explores requirements, system architecture, and automation for implementing personalized business-to-consumer (B2C), business-to-business (B2B), and consumer-to-consumer (C2C) e-business solutions. The approach supports collaborative applications where end users need to exchange messages, co-ordinate schedules and support workflow applications through automated forms. These capabilities should work transparently across all of the user's computing platforms - on their PC at home, at work, at an Internet café, on a PDA, or on a cell.

The proposed solution architecture is an intelligent agent-based framework built on collaborative crossplatform messaging using constraint-based methods to automate and personalize the behavior of the system. This approach aims at delivering intelligent business solutions that significantly enhance the user's experience and simplify the system integration effort. The solution employs a Java-based, intelligent agent architecture where software agents use constraints to streamline or possibly optimize such cross-platform requirements.

The proposed constraint satisfaction approach makes use of personalization data and content attributes to formulate the decisions needed to support effective collaboration among end users and business applications. Constraints are being used to help users arrange meetings, to route incoming messages to the most appropriate

Readers with comments or questions are encouraged to contact the author via email. 
receiving device (e.g. cell phone, email, PDA), and to assist users in the auto-completion of on-line forms such as purchase orders, agreements and invoices. The use of templates to simplify user entry and processing of constraints is being explored.

\section{Project Background}

The current project is a collaborative effort between The Wise Net Inc. (Dr. Honorio Todino), the Technical University of British Columbia (Dr. Toby Donaldson) and Oregon State University (author). The British Columbia Advanced Systems Institute (through the BC Advanced Systems Foundation), the Canadian National Research Council (through the Industrial Research Assistance Program) and The Wise Net are jointly financing the development and research efforts described in this paper. The research team supporting this effort is leveraging the following collective experience:

- $\quad$ Stock quotations pushed to pagers \& cell phones in real-time;

- $\quad$ Wireless development environments for wireless application protocol (WAP);

- $\quad$ Previous research into application of Instant Messaging (IM) and Short Messaging Service (SMS);

- $\quad$ Agent architectures using Javaspace technology (part of the Jini project);

- $\quad \mathrm{XML}, \mathrm{SOAP}$, and various distributed system architectures.

\section{Who Needs Personalization and Constraints?}

Our initial market assessment shows that the following types of users would benefit from personalization and automation features, namely, those who:

- $\quad$ Exchange large volumes of diverse transactions;

- $\quad$ Need to discriminate among transactions quickly and efficiently to react to business opportunities;

- $\quad$ Require relevant business data, reminders and notices in real-time;

- $\quad$ Are in a high cost business sector - their time is very valuable;

- Are constantly on the move with prolific computing needs: at the office, in the home and while in transit;

- Have non-trivial schedule coordination needs scheduling meetings with a constantly changing set of collaborating colleagues and customers;

- Would benefit from the ability of recording customer and other field data while in transit.

The following primary target markets have been identified:

- $\quad$ Real Estate Industry

- $\quad$ Home Care Industry

- $\quad$ Recruitment Industry

- $\quad$ Newspaper Classifieds

- $\quad$ Customer Relationship Management Market.

\section{What is Personalization?}

Personalization in many ways is a loaded term and needs to be somewhat demystified. Customization and adaptation are related terms that also need to be clarified. It can be safely said that these terms are all focused on improving the user's experience. In the e-business context this most often translates into enhancing customer satisfaction thereby increasing customer loyalty and repeat business. Useful perspectives on personalization may also be found in [3] and [4].

The Personalization Consortium [2] is a good source for defining personalization. For the purposes of the current work, however, it is necessary to go beyond their definitions to better relate personalization to the challenges of our problem set. We have therefore chosen to define a refined information network model for our purposes. 


\section{Information Network Model: "The Wise Net"}

The starting point for our personalization approach is Instone's personalization model described in [5]. With reference to user attributes and content attributes, it takes the position that personalization is achieved by discovering or defining business rules (a.k.a. personalization rules) that relate user and content attributes. However, there appear to be a few limitations to this model as outlined:

- Users collaborate with each other but there is no indication that they might collaborate with each other;

- $\quad$ It is not clear how services are addressed in this approach;

- $\quad$ The implications of collaborating through various network channels is not evident;

- $\quad$ The mapping of B2B, B2C and C2C e-business models is not obvious.

To address these limitations, we have defined an information network model called "The Wise Net" (TWN). The TWN model helps explain personalization and how intelligent agents implement constraint-based methods to support provide personalization, customization and adaptation capabilities.

The TWN model defines "content" as encompassing both goods and services available on or through the information network. And goods may be "physical products" or "information and data".

Users connected directly with each other over the network via messaging channels are said to be "communicating". Communicating users who also exchange goods are "collaborating". A user accessing an enterprise application is interacting with that application and thereby receiving goods and services (content).

"Network" usually implies the converged wired and wireless web but could also be referring to more limited contexts including local area networks, intranets, extranets, virtual private networks, wired-only networks, wireless local area networks, cellular-only networks and so forth.

Users will generally communicate through various devices using a number of messaging mechanisms. The concept of a logical communications channel ("channel" for short) has been defined. This includes the identification of the user device (PC, laptop, PDA, cell phone, fax,) and a messaging protocol (e-mail, instant messaging, short messaging service, wireless application protocol,). The TWN model will be extended to accommodate other modes of messaging as they emerge and become practical to integrate into the model.

Users may include groups of users, communities of users and businesses. A business is generally organized to deliver content, however, a user or group of users may also deliver goods and/or services (friendly advice and music for example).

To further generalize the model and support a symmetric view of the information network, it is useful to distinguish between information "consumers" and information "providers". One usually thinks of a business on the Internet as a "provider" of content and/or services. However, the supply-chain model reminds us that businesses also procure from upstream providers - typically, businesses are both "consumers" and "providers".

Conversely, users are normally thought of as "consumers". However, it is clear that they too may often take on the "provider" role. In fact the growth of open source peer-to-peer computing is rapidly creating new markets where ordinary users and groups of users are trading services and goods among themselves. Hence it is more general to characterize any user with the potential of behaving both as a "provider" and a "consumer".

This information network model, then, permits one to characterize both the provider and consumer aspects of B2B, B2C and C2C models. It also underscores the realization that these traditional e-business terms are actually

\footnotetext{
${ }^{1}$ In other documents, we have referred to "The Wise Net Framework" to describe the implementation of this model. We trust that there will be little or no confusion between these two terms - the TWN model is conceptual while the TWN framework is implementation specific.
} 
various perspectives or views of e-business behavior and do not, in fact, fully characterize any particular e-business operation.

The Wise Net model allows us to abstractly represent users, groups of users (communities) and businesses collaborating with each other, accessing services and exchanging physical goods and information/data (content). And this model assumes that consumers and providers are able to collaborate through a wide variety of messaging channels.

The final distinguishing feature is that consumers and provider personalization, customization and adaptation behaviors are enabled through a network of collaborating intelligent (more on this below).

\section{Personalization Defined}

This information network model, then, helps arrive at a more precise definition and scope for the term "Personalization".

At the highest level, personalization can be simply described as a process that facilitates interaction such that consumers and providers are able to more easily and more effectively access the content they desire or wish to deliver.

At a more specific level, personalization is achieved through a combination of effective interface design; customization and tailoring of interfaces and channels; automation of information and services to simplify and accelerate access; extraction of user preferences and constraints via observation and usage analysis; and inferencing of user preferences and constraints from the observed behaviours of others.

These definitions confirm that personalization maximizes relevancy and usability for both the consumer and the provider. However, the provider is usually motivated by customer (consumer) satisfaction and loyalty to select the "best buy" or use the highest quality communications channel. Of course the desires of consumers and providers may not always be compatible. For example, the consumer may prefer using a given channel to get timely access, but the provider may use another channel because it is cheaper. Similarly, a provider may want to offer the highest margin product, but the consumer may be more interested in a cheaper low margin product. These personalization conflicts need to be handled and resolved by the personalization system of course. It can be expected that the personalization process will also have a mediation role when the objectives of consumers and providers conflict.

Consumer and provider attributes (in particular "desires") are usually expressed as preferences or constraints (also referred to as objectives, goals, needs, and interests). Constraints are usually thought of as conditions that cannot be violated, while preferences are conditions that should be, but not necessarily, satisfied. Objectives, goals, needs, and interests can be expressed as preferences or constraints.

Personalization can take on the following forms:

- $\quad$ Customization of the look and feel of the consumer's user interfaces to make them as familiar and easy to use as possible in accordance with personal preferences and constraints;

- $\quad$ Selecting or adapting the consumer's communication channels to provide goods and services when and where required according to personal preferences and constraints;

- $\quad$ Making available only relevant goods and services in accordance with preferences and constraints specified by the consumer and/or the provider;

- Making available only relevant goods and services in accordance with the preferences and constraints of another consumer (user, group of users or business).

Customization refers to simple personalization implementations where the consumer explicitly (manually) specifies personalization data. This data is then used to programmatically configure user interfaces, select device channels and filter content sources. Adaptation refers to the ability of the system to observe consumer behavior to 
derive personalization data that is then used to configure user interfaces, select device channels and filter content sources. Collaborative filtering correlates a consumer's specified preferences and constraints to those of another consumer (or group of users) and filters content in accordance with that other consumer's observed preferences and constraints.

\section{What Are Constraints and Preferences?}

Formally, a constraint is simply a logical relation among two or more variables, each variable taking on a value in a given domain [6]. These domains are typically, but not necessarily, enumerated and finite sets of integers, characters, character strings, or symbolic names. A constraint restricts the values that a given variable can take on. Unary constraints are simple ones restricting the domain for a given variable. Binary constraints specify a relationship between two variables. N-ary constraints specify a relationship between more than two variables. An n-ary constraint can generally be expressed as a set of binary constraints. As mentioned above, preferences can be interpreted as "soft" constraints, that is, they are elastic constraints that can be relaxed when endeavoring to solve a constraint satisfaction problem.

Personalization problems involve characterizing some, but not all, provider and consumer attributes as constraints or preferences. A constraint problem consists of studying the values that these constrained / preferred variables could take on and discovering all sets of values that satisfy all of the constraints, and as many of the preferences as possible. This constraint solving process may discover one, many or no solutions. By allocating weights to the preferences, it should be possible to rank the solutions. If too many solutions are found, it may be possible to ask the consumer or provider to specify or search for additional constraints. If no solutions are found, the user can be asked to relax some of the constraints (convert them into preferences).

\section{Constraint Scenarios}

Let us consider a few simple collaborative scenarios to illustrate these ideas. Consumer Bob and provider Alice are real estate agents who communicate via their PCs, PDAs and cell phones and maintain calendars of their current activities distinguishing between private showings, public meetings, office hours, meals, special private events, sleep time and in transit (car / trip) time. They also exchange the following types of messages: info, new listing alerts, meeting reminders, contract forms and sales agreement notices ("agreement of sale has been signed!"). These variables could be represented as follows:

Devices $=\{\mathrm{pc}, \mathrm{pda}$, cell $\}$

- $\quad$ Channels $=\{$ email, instant messaging (im), short messaging service (sms) $\}$

- $\quad$ Activities $=\{$ showing, meeting, office, meal, event, sleep, transit $\}$

- $\quad$ Messages $=\{$ info, alert, reminder, form, contract, sale $\}$

Sample constraint / preference scenarios for Bob and Alice may be expressed as follows:

\section{Bob and Mary:}

- $\quad$ Send all messages to my e-mail account so that I can access all my messages through my pc when I am in my office or at home.

- When I am on my pc in the office or on my pc at home, send all listing alerts, meeting reminders and sale notices via instant messaging (im).

- When I am at a showing, meeting, enjoying a meal or in transit, send all listing alerts, meeting reminders and sale notices via SMS to my cell phone.

- Bob: When Mary sends me any type of message, I would prefer that it be sent to my e-mail rather than my cell phone.

- Mary: When I complete a new sale, send Bob a sales notice to his cell via SMS under all conditions.

- $\quad$ (Mary's constraint partially conflicts with Bob's preference - this will have to be mediated.) 


\section{Intelligent Agents}

An intelligent agent framework is very practical and effective for implementing personalization of the type being addressed by this project. Such a framework supports intelligent agents capable of performing specialized functions and collaborating with each other over a distributed information network solving various global tasks. The Alize project [7] is an interesting example of such an agent-based architecture for personalization.

The Wise Net's intelligent agent architecture has been implemented on top of Sun's Javaspace technology [8] - part of the Jini project [9]. TWN has developed specialized agents for interfacing to e-mail - POP3, IMAP, and SMTP to instant messaging systems - AIM, MSN Messenger, Yahoo! Messenger, and ICQ, and to cell phones Short Messaging Service (SMS) and Wireless Application Protocol (WAP). The framework implements middleware that integrates readily with a wide range of enterprise applications and web services.

In the approach under development, intelligent agents will implement constraint solvers to work on behalf of other agents. Specialized intelligent agents will call on the constraint solving agents to automate and personalize channel selection, forms filling, scheduling and message notification services (alerts and reminders).

\section{Current Project Status and Plans}

The project is currently developing a constraint engine prototype that it plans to demonstrate by the spring of this year. Meanwhile, the TWN model and framework continue to be refined. A market study aimed at narrowing the desired functions and features of the target markets is nearing completion. In parallel, a field trial has been launched in the real estate industry to validate specific personalization and automation requirements of that market segment. Together, the market study and field trial will shape the personalization strategy that will be fielded in the initial product release late next summer.

The current phase of the project is focusing on basic intelligent agent and personalization features automating channel selection, calendaring, and forms handling. A follow-on project is planned to produce a more advanced intelligent agent architecture with more intensive focus on assessing its scalability and performance properties.

\section{References}

1. K.C. Toth and H. Todino, Instant Internet Intelligence for Wireless Business Applications, International Applied Business Research Conference, Cancun Mexico, March 2001

2. The Personalization Consortium, http://www.personalization.org/personalization.html

3. Jacob Nielsens' Alertbox for October 4, 1998: Personalization is Over-Rated, http://www.useit.com/ alertbox/981004.html

4. Bonett, Monica, Personalization of Web Services: Opportunities and Challenges, UKOLN, University of Bath, June 22, 2001, http://www.ariadne.ac.uk/issue28/personalization/intro.html

5. Instone, K., Information Architecture and Personalization, Argus Associates, December 2000, http://argusacia.com/white_papers/personalization.html

6. Bartak, Roman, Guide to Constraint Programming, 1998, http://kti.ms.mff.cuni.cz/ bartak/ constraints/intro.html

7. Cerquides, J. et. Al., Alize: An Agent-Based Architecture for Personalization, Intelligent Software Components, http://www.isoco.com

8. The Source for Java Technology, http://java.sun.com/products/javaspaces/

9. Jini Network Technology, http://www.sun.com/jini/ 